\title{
Ureteral access sheath during retrograde flexible uretero-renoscopy: safe tool or safety hazard?
}

\author{
Răzvan Mulțescu, Bogdan Geavlete, Răzvan Satalan, Dragoș Georgescu, Petrișor Geavlete
}

Corresponding author:

Razvan Multescu MD, PhD

Department of Urology,

Saint John Emergency Clinical Hospital

Vitan-Barzesti 13, Sector 4, 042122 ,

Bucharest, Romania

E-mail: razvanmultescu@yahoo.com
Department of Urology, Saint John Clinical Emergency Hospital Bucharest, Romania

\section{ABSTRACT}

Objectives: To evaluate the morbidity of ureteral access sheath (UAS) use during retrograde flexible ureteroscopy, and after a minimum follow-up period of 18 months.

Methodology: We prospectively evaluated 237 patients treated by retrograde flexible ureteroscopic approach for single pyelocaliceal stones: 126 in which a 10/12F UAS was used were compared with 111 in which the procedure was performed without it. Patients were followed for at least 18 months. $15 \%$ of the first group and $12.6 \%$ of the second one were $\mathrm{JJ}$ stented preoperatively. UAS related ureteral wall injuries we recorded in $36.5 \%$. According to Traxer classification, $32.5 \%$ were grade 1 injuries, $3.2 \%$ were grade 2 injuries and $0.8 \%$ grade 3 injuries. Perioperative complications rate were similar among the two groups $(11.1 \%$ vs. $14.4 \%$ ), septic ones being significantly reduced in the study group (28.3\% vs. $68.7 \%$ out of the total complication cases. 22 patients were lost for follow-up. At 18 months one case with stenosis of the ureteral orifice was encountered in the control group.

Conclusions: Prudent use of UAS does not increase the complication' rates specific for the flexible ureteroscopic approach. It seems to be associated with less septic complications, probably by maintaining a low pressure in the collecting system.

Key words: ureteral access sheath, morbidity, flexible ureteroscopy, urolithiasis

\section{INTRODUCTION}

Ureteroscopy is nowadays a widespread procedure in endourologic centers. Despite the long period of employment of these interventions and the large amount of data gathered and published, there are still some controversial issues.

The use of a ureteral access sheath and stenting habits are probably the most disputed elements. Some pro-access sheath arguments as well as against its use were advocated by various authors.

The aim of our study was to evaluate in a prospective manner the differences between the routine use of ureteral access sheath vs. no use of such a device in cases of pyelocaliceal stones. 


\section{MATERIAL AND METHODS}

We prospectively evaluated 237 patients treated by retrograde flexible ureteroscopic approach for single pyelocaliceal stones between 1 and $2 \mathrm{~cm}$ in size.

The cases were divided in two groups: the study one, with 126 patients in which a Cook Flexor 10/12F ureteral access sheath was used, and the control one of 111 patients in which the procedure was performed without such an ancillary instrument. In all cases, a Storz Flex-Xc flexible ureteroscope and Holmium laser lithotripsy were applied.

If any difficulties were encountered during the ureteral access sheath indwelling, the procedure was stopped, a JJ stent was inserted and another intervention was attempted after 1-2 weeks.

Intraprocedural visibility was subjectively assessed, being scored by the operator from 1 to 5 (1-very poor, with the potential to impose termination of the procedure, 2-poor, 3-average, 4-good, 5-excellent). At the end of the procedure, the endoscope was extracted en bloc with the ureteral access sheath while also performing a thorough visual inspection of the ureter.

Pre-ureteroscopy stenting necessities, intraprocedural characteristics, stone-free rates and complications stratified in Clavien classification were evaluated for each group and compared.

The statistical analysis of various parameters of the two study arms was performed using standard statistical software (IBM SPSS Statistics v20, Chicago, IL).

\section{RESULTS}

$9 / 126(7.1 \%)$ of the patients in the first group and $11 / 111$ (9.9\%) in the second one were already JJ stented when the retrograde flexible ureteroscopic approach was attempted (Chi-Square test, $p=0.44$ ). Due to difficulties to ascend the ureteral access sheath, a supplementary $10 / 126(7.9 \%)$ of the cases from the study group were also pre-stented, while in the second group, impossible ureteral passage of the flexible ureteroscope imposed a JJ stent indwelling in 3/111 (2.7\%) of the patients (Chi-Square test, $\mathrm{p}=0.07$ ).

In order to eliminate any procedural related biases, Holmium laser lithotripsy to dust was employed in all cases. The mean operative times were similar between the two groups (45 versus 41 minutes). The stone-free rates after one procedure were similar, $123 / 126$ (97.6\%) in the study group versus 108/111 (97.3\%) in the second one.

The intraprocedural visibility was significantly better when using the ureteral access sheath (with a mean score of 4.51) by comparison to the interventions performed without it (mean score of 3.82) (Student T-test, $p<0.001$ ).

The perioperative complication' rates were similar among the two groups (Chi-Square test, $p=0.44$ ), most of them being minor ones.

Ureteral access sheath related ureteral wall injuries we recorded in 46/126 (36.5\%). According to Traxer classification (1), 41/126 (32.5\%) were grade 1 injuries, $4 / 126(3.2 \%)$ were grade 2 injuries and 1/126 (0.8\%) had grade 3 injuries. No grade 4 injuries occurred.

When we evaluated the complications' stratification according to the Clavien System, we noticed that the severity of those in the control group was slightly higher than in the study group, however, the difference not being statistically significant (Student $T$-test, $p=0.17$ ) (table 1).

Septic complications (from prolonged fever to sepsis) represented $4 / 14(28.3 \%)$ of the complications occurring in the first group, significantly less by comparison to $11 / 16(68.7 \%)$ in the control one (ChiSquare test, $p=0.034$ ).

110 patients in the first group and 105 in the second one were followed-up for at least 18 months. At 18 months one case with stenosis of the ureteral orifice was encountered in the control group.

\section{DISCUSSION}

The use of the ureteral access sheath still represents a controversial issue. To prove this ongoing debate, during the Societe Internationale d'Urologie International Consultation on Urological Diseases (SIU-ICUD) Joint Consultation on Stone Disease (presented in Glasgow, during the 2014 Congress of SIU and which will be published in 2015) the Committee on "Stone Technology:URS" does not recommend the routine use of ureteral access sheath, while the Committee on "Antibiotics and Handling and Prevention of Complications" recommend it as one of the two alternatives to prevent septic complications (2).

The frequency of ureteral access sheath employ-

Table 1 - Complications rates in the two studied groups

\begin{tabular}{lcc}
\hline & Group I & Group II \\
\hline Complications rate & 14 & 16 \\
\hline Clavien I complications & 8 & 4 \\
\hline Clavien II complications & 5 & 7 \\
\hline Clavien III complications & 1 & 4 \\
\hline Clavien IV complications & 0 & 1 \\
\hline Clavien V complications & 0 & 0 \\
\hline
\end{tabular}


ment in the published data varies significantly. Delfidio et al. reported the use of such instruments in $25 \%$ of patients, Traxer et al. in 58\% while Miernik and coworkers described a standardized flexible ureteroscopic technique and used it in $71 \%$ of cases $(3,4,5)$.

The partisans of this device advocate the fact that it offers an easy access to the upper urinary tract as many times as necessary, maintains a low pressure in the collecting system during the procedure and it may increase the flexible ureteroscopes' lifespan by reducing the resistance during insertion and consequently the stress at the tip of the instrument $(3,5)$. Reduced pressure in the collecting system may prevent pyelovenous and pyelolymphatic reflux, translating into clinical benefits during retrograde ureteroscopic approach of upper-tract transitional cell carcinoma, struvite stones, or calculi associated with urinary tract infection (6).

In our study, the backflow between the flexible ureteroscope and the sheath offered a better visibility and it may also explain the reduced incidence and severity of the septic complications in the study group.

However, there are also arguments against the routine use of ureteral access sheaths such as increased intra- or postoperative morbidity as well as adding supplementary costs for an already expensive procedure (7).

Ureteral lesions following the use of such a device were studied by Traxer and Thomas, who identified wall injury in $46.5 \%$ of the cases, severe ones involving the smooth muscle layers being observed in $13.3 \%$ of the cases. The most significant predicting factor of ureteral injuries was the absence of previous ureteral stenting, others being male sex and older age (1). In our study, severe lesions involving the muscular layer were encountered in only $4 \%$ of the patients, with preservation of the adventitial layer in 4 cases and periureteral fat seen in only 1 case. We strongly believe that careful case selection, preoperative stenting in patients that require passive dilation and gentle indwelling of the ureteral access sheath are the key elements in preventing significant ureteral wall damage.

Regarding the potential of the ureteral access sheath to generate ureteral strictures due to the ischemic effect on the ureteral wall or other long term complications, a thorough evaluation of the literature doesn't offer strong data supporting such a hypothesis. Although possible, it is not a frequent occurrence, being probably related more often with ureteral wall trauma, impacted stones and/or other individual factors rather than ureteral access sheath use $(8,9,10)$. More over, a study by Delvecchio on 71 cases in which larger size sheaths were used $(12 / 14 \mathrm{~F}$ in $78.9 \%$ of the cases and $14 / 16 \mathrm{~F}$ in $9.8 \%$ of the cases) only one stricture was identified on follow-up imaging (1.4\%), apparently not related to the use of this device (11). In our study only one case with stenosis of the ureteral orifice was encountered at 18 months follow-up, in the control group.

Evaluating the costs, the published data seems to favor ureteral access sheath, although cost-effectiveness analysis in large, randomized, prospective studies is required (12).

Supplementary spending for this device may be compensated by increasing durability of flexible ureteroscopes due to reduced stress at the tip during insertion or by a decreased damage risk by backloading a stiff guide wire (as needed when the sheath is not employed) $(5,10)$. However, these hypotheses require further studies. Overall costs may also be diminished by reducing operative time and, if achievable, by preventing severe intra- or postoperative complications $(10,13)$.

As a precautionary measure, employment for a longer period of time of unnecessary large sheaths should be avoided (11). In this regard, a study of Ng and coworkers demonstrated that increased access sheath diameter does not improve flow when the working channel of the flexible ureteroscope is occupied with ancillary instruments (14). The only advantage of a larger sheath remains accommodation of larger extractable stone fragments. For this reason, thinner sheaths may be employed still obtaining all the other favourable effects, without significant risks.

In addition to facilitating ureteroscopic access, reducing costs, and lowering intrarenal pressures, there are papers reporting even improvements of stone free rates when such devices were used (15). However, in our study the efficacy proved to be similar in both series.

\section{CONCLUSIONS}

Routine but careful use of ureteral access sheath does not increase the complication' rates specific for the flexible ureteroscopic approach. Moreover, the severity of these adverse events seems to be reduced by comparison to patients in which such a device was not used.

It offers some clear advantages regarding access, visibility and seems to be associated with less septic complications, probably by maintaining a low pressure. Also, late complications such as ureteral stricture didn't correlate with ureteral access sheath use in our series. 
The real financial burden associated with the routine use of such a device is still to be evaluated.

\section{Acknowledgment}

This work received financial support through the project "CERO - Career profile: Romanian Researcher", grant number POSDRU/159/1.5/S/135760, co-financed by the European Social Fund for Sectorial Operational Program Human Resources Development 2007-2013.

\section{REFERENCES}

1. Traxer $\mathbf{0}$, Thomas A. The prospective evaluation and classification of ureteral wall injuries resulting from insertion of a ureteral access sheath during retrograde intrarenal surgery. J Urol 2013; 189:580-584

2. academy. siu-urology.org/siu/\#!*menu $=16^{*}$ browseby $=2 *$ sortby $=$ $1 *$ media $=1 *$ label $=3930$

3. Defidio L, De Dominicis M, Di Gianfrancesco L, Fuchs G, Patel A. Improving flexible ureterorenoscope durability up to 100 procedures. J Endourol 2012; 26:1329-1334

4. Miernik A, Wilhelm K, Ardelt PU, Adams F, Kuehhas FE, Schoenthaler M. Standardized flexible ureteroscopic technique to improve stone-free rates. Urology 2012; 80:1198-1202

5. Traxer 0, Dubosq F, Jamali K, Gattegno B, Thibault P. New-generation flexible ureterorenoscopes are more durable than previous ones. Urology 2006; 68:276-281
6. Auge BK, Pietrow PK, Lallas CD, Raj GV, Santa-Cruz RW, Preminger GM. Ureteral access sheath provides protection against elevated renal pressures during routine flexible ureteroscopic stone manipulation. J Endourol 2004 Feb;18(1):33-6

7. Abrahams HM, Stoller ML. The argument against the routine use of ureteral access sheaths. Urol Clin North Am 2004; 31:83-87

8. Tas S, Tugcu V, Mutlu B, Karadag S, Bitkin A, Yücel M, Tasçi Al. Incidence of ureteral stricture after ureterorenoscopic pneumatic lithotripsy for distal ureteral calculi. Arch Ital Urol Androl. 2011; 83(3):141-6

9. Wang HH, Huang L, Routh JC, Kokorowski P, Cilento BG Jr, Nelson CP. Use of the ureteral access sheath during ureteroscopy in children. J Urol. 2011; 186(4 Suppl):1728-33

10. Vanlangendonck R, Landman J. Ureteral access strategies: pro-access sheath. Urol Clin North Am. 2004; 31(1):71-81

11. Delvecchio FC, Auge BK, Brizuela RM, Weizer AZ, Silverstein AD, Lallas CD, Pietrow PK, Albala DM, Preminger GM. Assessment of stricture formation with the ureteral access sheath. Urology. 2003; 61(3):518-22

12. Stern JM, Yiee J, Park S. Safety and efficacy of ureteral access sheaths. J Endourol. 2007; 21(2):119-23

13. Kourambas J, Byrne RR, Preminger GM. Does a ureteral access sheath facilitate ureteroscopy? J Urol. 2001; 165(3):789-93

14. Ng YH, Somani BK, Dennison A, Kata SG, Nabi G, Brown S. Irrigant flow and intrarenal pressure during flexible ureteroscopy: the effect of different access sheaths, working channel instruments, and hydrostatic pressure. J Endourol. 2010 Dec;24(12):1915-20

15. L'Esperance JO, Ekeruo WO, Scales CD Jr, Marguet CG, Springhart WP, Maloney ME, Albala DM, Preminger GM. Effect of ureteral access sheath on stone-free rates in patients undergoing ureteroscopic management of renal calculi. Urology. 2005 Aug;66(2):252-5 